\title{
ON INFINITESIMAL AFFINE AND ISOMETRIC TRANSFORMATIONS PRESERVING RESPECTIVE VECTOR FIELDS
}

\author{
By TOSHIHIRo IWAI
}

\section{$\S 1$. Introduction.}

Researches into symmetry of dynamical systems exhibit an increase of interest in automorphisms of geometric objects with some dynamical constraints. Recently, Ikeda and Nishino [1] have studied scalar-preserving isometries of a Riemannian space. In 1968-1974, Katzin and Levine treated projective and conformal transformations related with dynamical structures [2], [3], [4], [5]. The present author [6] has shown that an invariance of the equations of motion in a Riemannian space entails an affine or isometric transformation which preserves a vector field. Interest in the same kinds of transformations comes also from other branch [7]. This paper presents solutions to equations proposed in the previous paper [6] and reveals what Riemannian spaces can admit invariance Lie algebras consisting of the infinitesimal affine and isometric transformations preserving respective vector fields. Global situations will be touched upon.

The settings of the problem are briefly reviewed in Section 2. Section 3 deals with Lie algebras of the infinitesimal affine transformations preserving a vector field. Maximal Lie algebras and the allowed Riemannian spaces will be found out there. Section 4 is concerned with Lie algebras of infinitesimal isometries which preserve a vector field, and with the problem of what Riemannian spaces can admit such Lie algebras. Global structures of these Riemannian spaces will be described. Section 5 is devoted to studying a Lie algebra of dimension lower than that of the considered ones in Section 4; this Lie algebra consists of the infinitesimal isometries preserving a scalar. A case which was out of consideration in [1] will be studied. It will be shown what Riemannian spaces can admit Lie algebras of the infinitesimal isometries preserving a scalar, together with remarks on global situations.

Received September 24, 1976. 


\section{$\S 2$. Basic equations.}

Let $(M, g)$ be an $n$-dimensional Riemannian space ${ }^{1)}$, and $\left(x^{i}\right)^{2)}$ a local coordinate system of $M$. Let $T(M)$ be the tangent bundle over $M$ and $\left(x^{i}, v^{i}\right)$ the induced coordinate system of $T(M)$. The geodesic spray is a vector field on $T(M)$ given by

$$
E=v^{2}\left(\frac{\partial}{\partial x^{2}}-\left\{\begin{array}{c}
j \\
i k
\end{array}\right\} v^{k} \frac{\partial}{\partial v^{j}}\right)
$$

where $\left\{\begin{array}{c}j \\ i k\end{array}\right\}$ denote Christoffel symbols formed from the metric $g$. Given a vector field $V$ on $M$ with local components $\left(V^{i}\right)$, define a vector field on $T(M)$ by

$$
Z=E+V^{i} \frac{\partial}{\partial v^{j}} \text {. }
$$

The field $Z$ is defined throughout $T(M)$. Integral curves of $Z$ describe time development of the dynamical system $(T(M), Z)$. An infinitesimal transformation $X=\left(\xi^{i}\right)$ of $M$ is naturally lifted to $T(M)$ by

$$
\tilde{X}=\xi^{2} \frac{\partial}{\partial x^{2}}+\frac{\partial \xi^{2}}{\partial x^{i}} v^{j} \frac{\partial}{\partial v^{2}} .
$$

Theorem 2.1 [6]. A necessary and sufficient condition for $Z$ given by (2.2) to admit an infinitesimal transformation $\tilde{X}$ given by (2.3), that is, $[\tilde{X}, Z]=0$, is

$$
\mathcal{L}_{X}\left\{\begin{array}{c}
i \\
j k
\end{array}\right\}=0, \quad \mathcal{L}_{X} V^{i}=0,
$$

where $\mathcal{L}_{X}$ denotes the Lie derivation with respect to $X$.

Alternatively, denote by $T^{*}(M)$ the cotangent bundle over $M$ with the induced coordinate system $\left(x^{i}, p_{i}\right)$. The vector field $Z$ on $T(M)$ is carried over, by the natural diffeomorphism of $T(M)$ with $T *(M)$, to

$$
Z^{\prime}=\frac{\partial T}{\partial p_{i}} \frac{\partial}{\partial x^{2}}-\frac{\partial T}{\partial x^{2}} \frac{\partial}{\partial p_{i}}+V_{\imath} \frac{\partial}{\partial p_{i}},
$$

where $T=\frac{1}{2} g^{i j} p_{i} p_{j}$ and $V_{j}=g_{j k} V^{k}$. For a given vector field $X$ on $M$, we put

$$
X^{*}=\xi^{2} \frac{\partial}{\partial x^{2}}-\frac{\partial \xi^{\jmath}}{\partial x^{2}} p_{j} \frac{\partial}{\partial p_{i}},
$$

which is the natural lift to $T^{*}(M)$.

1) All geometric objects in this paper are supposed to be of class $C^{\infty}$.

2) Unless otherwise stated, Latin indices run from 1 to $n$, and the summation convention is adopted. 
THEOREM 2.2 [6]. $Z^{\prime}$ given by (2.5) admits an infinitesimal transformation $X^{*}$ given by (2.6), if and only if

$$
\mathcal{L}_{X} g_{\imath j}=0, \quad \mathcal{L}_{X} V_{i}=0 .
$$

We are going to solve Eqs. (2.4) in Section 3 and Eqs. (2.7) in Section 4, respectively. We shall consider in Section 5 Eqs. (2.7) with gradient $V$ in detail.

\section{§ 3. Infinitesimal affine transformations.}

By $(S)$ we mean the Lie algebra of all the vector fields $X$ satisfying (2.4). To investigate $\mathbb{S}$ we put (2.4) into a normal form :

$$
\begin{gathered}
\nabla_{\imath} \xi^{j}=\xi_{i} \cdot{ }^{\jmath}, \quad \nabla_{j} \xi_{k} \cdot=-R_{l j k} \cdot \xi^{l}, \\
\xi^{k} \nabla_{k} V^{i}-V^{k} \xi_{k} \cdot=0,
\end{gathered}
$$

where $\left(R_{l j k}{ }^{i}\right.$. $)$ denotes the curvature tensor and $\nabla_{\imath}$ the covariant derivation with respect to $\partial / \partial x^{i}$. Eqs. (3.1) and (3.2) are linear differential equations in $n^{2}+n$ unknowns $\left(\xi^{i}, \xi_{,}{ }^{k}\right)$ with the constraints. We assume that the vector field $V=\left(V^{i}\right)$ is not the zero but may have isolated zero points.

THEOREM 3.1. Lie algebras of infinitesimal affine transformations preserving a non-zero vector field are of dimension $n^{2}$ at most. An $n^{2}$-dimensional algebra, when such exists, is transitive at every point where $V$ does not vanish.

Proof. By bringing (3.2) into the form

$$
\xi^{k} \nabla_{k} V^{i}-V^{k} \delta_{j}^{i} \xi_{k}^{\jmath}=0
$$

and by considering the rank of the $\left(n, n^{2}\right)$-matrix $\left(V^{k} \delta_{j}^{i}\right)$ in a local coordinate system such that $\left(V^{i}\right)=(1,0, \cdots, 0)$, we find that (3.2) contains at least $n$ linearly independent equations in $\left(\xi^{2}, \xi_{,}{ }^{k}\right)$. Hence Eqs. (3.1) and (3.2) admit at most $n^{2}\left(=n^{2}+n-n\right)$ linearly independent solutions. When $n^{2}$ independent solutions are admitted, by means of (3.3) the initial values of $\left(\xi^{i}\right)$ can be arbitrarily chosen at each point where $V$ does not vanish, and consequently $(S)$ is transitive. This completes the proof.

By straightfoward calculation we can conclude that if the integrability conditions

$$
\xi^{m} \nabla_{m} R_{\imath j k} \cdot+\xi_{\imath}{ }^{m} R_{m j k} \cdot+\xi_{\jmath} \cdot R_{\imath m k} \cdot+\xi_{k} \cdot R_{\imath \jmath m} \cdot-\xi_{m}^{l} \cdot R_{\imath \jmath k} \cdot m=0
$$

of (3.1) are satisfied for any $\left(\xi^{\imath}, \xi_{\jmath}^{k}\right)$ satisfying (3.2) then $M$ is locally flat: $R_{\imath j k l}=0$. Thus, we have

THEOREM 3.2. If a Riemannian space $M$ admits a maximal Lie algebra of the infinitesimal affine transformations preserving a non-zero vector field, then it is locally flat. 
Although groups of affine transformations of an affinely connected space have been already investigated by Wang and Yano [8], our result about affine transformations preserving a non-zero vector field is not necessarily contained in theirs. For in our case singular points of transformations, which was out of consideration in [8], are allowed.

In what follows we shall work with (3.2) for $\mathbb{B}$ of maximal dimension $n^{2}$. By Theorem 3.2 $M$ is locally identified with $\mathbf{R}^{n}$ having the standard coordinate system $\left(x^{i}\right)$. Let $G$ denote a local Lie group generated by a maximal Lie algebra (S) and $G_{0}$, isomorphic with a subgroup of the general linear group $\operatorname{GL}(n, \mathbf{R})$, the isotropy subgroup of $G$ at a point of $M$. Then we have

$$
n^{2}-n \leqq \operatorname{dim} G_{0} \leqq n^{2} .
$$

Since $\left(5\right.$ is transitive at every point where $V \neq 0$ (see Theorem 3.1), $G_{0}$ is of dimension $n^{2}-n$ at the points. If $V=0$ at some point, (3.3) holds identically for all $\left(\xi^{2}, \xi_{\jmath}{ }^{k}\right)$ corresponding to the elements of the Lie algebra $\mathbb{G}_{0}$ of $G_{0}$ and hence gives no constraints, so that $G_{0}$ is of dimension $n^{2}$ there. Thus we get

$$
\operatorname{dim} G_{0}=n^{2} \text { or } n^{2}-n,
$$

according as $V$ vanishes at a point or not.

About subgroups of $\mathrm{GL}(n, \mathbf{R})$ the following Lemma 3.3 due to Wang and Yano [8] is available. We use here the following notations:

$$
\begin{aligned}
\mathrm{GL}^{+}(n, \mathbf{R}) & =\left\{\left(a^{2}{ }_{\jmath}\right) ;\left|a^{2}{ }_{j}\right|>0\right\}, \\
L & =\left\{\left(a^{2}{ }_{\jmath}\right) ; a^{1}{ }_{1}=1, a^{\lambda}{ }_{1}=0,\left|a^{2}{ }_{j}\right|=1\right\}^{3)}, \\
L^{\prime} & =\left\{\left(a^{2}{ }_{\jmath}\right) ; a^{1}{ }_{1}=1, a^{1}{ }_{\lambda}=0,\left|a^{2}{ }_{j}\right|=1\right\}, \\
M & =\left\{\left(a^{2}{ }_{\jmath}\right) ; a^{1}{ }_{1}>0, a^{2}{ }_{1}=0,\left|a^{2}{ }_{j}\right|=1\right\}, \\
M^{\prime} & =\left\{\left(a^{2}{ }_{\jmath}\right) ; a_{1}{ }_{1}>0, a^{1}{ }_{\lambda}=0,\left|a^{2}{ }_{j}\right|=1\right\}, \\
K & =\left\{\left(a^{2}{ }_{j}\right) ; a^{\imath}{ }_{j}=a \delta^{2}{ }_{j}, a: \text { positive real }\right\}, \\
I(b) & =\left\{\operatorname{diag}\left(e^{(1+b) t}, e^{b t}, \cdots, e^{b t}\right) ; b \text { : fixed real }\right\},
\end{aligned}
$$

where $t$ is the parameter of the group $I(b)$. In this notations the lemma is stated as follows.

LEMMA 3.3. Let $H$ be a closed and connected subgroup of $\mathrm{GL}(n, \mathbf{R})$. If $\operatorname{dim} \mathrm{H} \geqq n^{2}-2 n+5$, then $H$ is conjugate to one of the groups: $\mathrm{GL}^{+}(n, \mathbf{R})$, $\mathrm{SL}(n, \mathbf{R})$ (the special linear group), $K \times L, K \times L^{\prime}, K \times M, K \times M^{\prime}, I(b) \times L$, $I(b) \times L^{\prime}, L, L^{\prime}$.

3) Greek indices range over $2,3, \cdots, n$. 
For $n \geqq 5$ Lemma 3.3 provides all possible subgroups $G_{0}$ of dimension greater than or equal to $n^{2}-n$. Accordingly, we can determine a vector field preserved by an isotropy subgroup $G_{0}$. Because of (3.4) we single out $n^{2}$ - and $\left(n^{2}-n\right)$ dimensional subgroups of $\mathrm{GL}^{+}(n, \mathbf{R})$ as follows: $\mathrm{GL}^{+}(n, \mathbf{R}), K \times L, K \times L^{\prime}$, $I(b) \times L$ and $I(b) \times L^{\prime}$. Thus in our situation $\mathbb{S}_{0}$ is necessarily one of the Lie algebras of these subgroups. We now adress ourselves to determining preserved vector fields $V$ by solving (3.2) for each Lie algebra $\mathbb{S}_{0}$. After doing so, we consider what infinitesimal affine transformations other than the elements of $\mathbb{S}_{0}$ can preserve (i. e., be commutative with) the already determined vector field $V$.

(i) $G_{0}=\mathrm{GL}^{+}(n, \mathbf{R}) . \quad \mathrm{A}$ basis of $\mathscr{G}_{0}$ on $M$ consists of ${ }^{4)}$

$$
x^{2} \frac{\partial}{\partial x^{j}} \text {. }
$$

Substituting (3.5) for $\left(\xi^{i}\right)$ into (3.2) shows that the vector field $V$ commutative with (3.5) is

$$
V=c x^{2} \frac{\partial}{\partial x^{2}} \quad(c \neq 0: \text { constant }) .
$$

It is easily seen that (3.6) is not commutative with any infinitesimal affine transformations other than (3.5). Thus $G=G_{0}$, and hence $\operatorname{dim} \mathscr{S}=n^{2}$.

(ii) $G_{0}=K \times L$. After the same method as above, we can prove that $V$ has the same form as (3.6) in this case. It is an easy matter to see that if $V$ is commutative with an arbitrary infinitesimal affine transformation other than the elements of $\mathbb{S}_{0}$ then $V$ vanishes. Therefore $G=G_{0}$ holds, so that $\operatorname{dim} \mathbb{G}=n^{2}-n$.

(iii) $G_{0}=K \times L^{\prime}$. By substituting the elements of $\mathfrak{S}_{0}$ for $\left(\xi^{i}\right)$ into (3.2), we find that

$$
V=c x^{\lambda} \frac{\partial}{\partial x^{\lambda}} \quad(c \neq 0: \text { constant })
$$

is preserved by $\mathscr{G}_{0}$. An infinitesimal transformation $\partial / \partial x^{1}$ is the only one commutative with (3.7), as is easily seen. Thus, $G=G_{0} \times \mathbf{R}$ and $\operatorname{dim} \mathbb{B}=n^{2}-n+1$.

(iv) $G_{0}=I(b) \times L . \quad$ A basis of $\mathbb{S}_{0}$ consists of

$$
(1+b) x^{1} \frac{\partial}{\partial x^{1}}+b x^{\lambda} \frac{\partial}{\partial x^{\lambda}}, \quad x^{\lambda} \frac{\partial}{\partial x^{1}}, \quad x^{\lambda} \frac{\partial}{\partial x^{\mu}}-\frac{1}{n-1} \delta_{\mu}^{\lambda} x^{\kappa} \frac{\partial}{\partial x^{\kappa}} .
$$

A vector field commutative with any element of $\mathbb{B}_{0}$ turns out to be

$$
V= \begin{cases}c x^{2} \frac{\partial}{\partial x^{2}}+c^{\prime} \frac{\partial}{\partial x^{1}} & \text { for } 1+b=0, \\ c x^{2} \frac{\partial}{\partial x^{2}} & \text { for } 1+b \neq 0,\end{cases}
$$

4) Without loss of generality we can suppose that $G_{v}$ is the isotropy group at the origin $\left(x^{i}\right)=0$. 
where $c$ and $c^{\prime}$ are constants. Furthermore, infinitesimal affine transformations $\partial / \partial x^{k}(k=1, \cdots, n)$ can preserve (3.9), when and only when $1+b=0$ and $c=0$. Hence, $G=G_{0} \cdot \mathbf{R}$ with $1+b=0$ and $\operatorname{dim} \$=n^{2}$, where the dot means the semidirect product.

(v) $G_{0}=I(b) \times L^{\prime}$. Following the method used above, we can see that the preserved vector field $V$ is of the form

$$
V= \begin{cases}c\left(x^{1}\right) x^{\lambda} \frac{\partial}{\partial x^{\lambda}} & \text { for } 1+b=0, \\ c x^{\lambda} \frac{\partial}{\partial x^{\lambda}} & \text { for } 1+b \neq 0,\end{cases}
$$

where $c\left(x^{1}\right)$ is a function of $x^{1}$ alone and $c$ is a constant. If (3.10) is commutative with an infinitesimal affine transformation other than the elements of $\mathbb{S}_{0}$, then $c\left(x^{1}\right)=c=0$. Hence, $G=G_{0}$ and $\operatorname{dim}\left(s=n^{2}-n\right.$.

Summarizing the above discussion, we obtain

THEOREM 3.4. If a Riemannian space of dimension $n(n \geqq 5)$ admits a maximal Lie algebra $\mathbb{S}$ of the infinitesimal affine transformations preserving a non-zero vector field, then the preserved vector field is either $c x^{2} \partial / \partial x^{2}$ or $c^{\prime} \partial / \partial x^{1} \quad\left(c, c^{\prime}\right.$ : consts.) in a suntable coordinate system. The admitted local group generated by $\mathbb{S}$ is locally isomorphic to $\mathrm{GL}^{+}(n, \mathbf{R})$ or $(I(b) \times L) \cdot \mathbf{R}^{n}$ with $1+b=0$, according to whether the preserved vector field is $c x^{2} \partial / \partial x^{2}$ or $c^{\prime} \partial / \partial x^{1}$.

Remark: If the Riemannian space is the Euclidean space $\mathbf{R}^{n}$, Theorem 3.4 can be easily set up in global situation.

\section{$\S 4$. Infinitesimal isometries.}

Let $\&$ denote the Lie algebra consisting of the infinitesimal isometries preserving a vector field; this is determined by (2.7). As is well known, (2.7) is equivalent to

$$
\begin{gathered}
\nabla_{\imath} \xi^{\jmath}=\xi_{i}{ }^{\jmath}, \quad \nabla_{k} \xi_{i} \cdot=-R_{l k i}{ }^{\jmath} \cdot \xi^{l}, \\
\xi_{\imath \jmath}+\xi_{j i}=0, \quad \xi^{k} \nabla_{k} V_{j}+V^{k} \xi_{j k}=0, \quad\left(\xi_{\imath j}=g_{j k} \xi_{\imath}{ }^{k}\right) .
\end{gathered}
$$

We assume that $V$ is not the zero but may have isolated zero points, as well as in Section 3. Eqs. (4.2), the set of constraints on $n^{2}+n$ unknowns $\left(\xi^{\imath}, \xi_{,}{ }^{k}\right)$, can be written as

$$
\xi^{k} \nabla_{k} V,+T,{ }^{A} \xi_{A}=0
$$

where $T_{j}{ }^{A}=\delta_{j}^{l} V^{k}, A=(l, k)$ with $l>k$. By choosing a local coordinate system such that $\left(V^{i}\right)=(1,0, \cdots, 0)$, we can see that the rank of the matrix $\left(T_{j}{ }^{A}\right)$ is 
equal to $n-1$. Accordingly, the linear differential equations (4.1) and (4.2) may admit at most $n(n-1) / 2+1\left(=n^{2}+n-n(n-1) / 2-(n-1)\right)$ linearly independent solutions. When the maximal number of independent solutions are admitted, by means of (4.3) the initial values of $\left(\xi^{i}\right)$ can be chosen arbitrarily, so that $\mathbb{B}$ is transitive at each point where $V$ does not vanish.

Let $G$ be a local isometry group generated by a maximal algebra $\mathbb{B}$ and $G_{0}$ an isotropy subgroup of $G$ at a point of $M$. Then

$$
\frac{1}{2}(n-1)(n-2) \leqq \operatorname{dim} G_{0} \leqq \frac{1}{2} n(n-1)+1 .
$$

If $V \neq 0, G$ is transitive, as is claimed above, so that $\operatorname{dim} G_{0}=(n-1)(n-2) / 2$. If $V=0$ at a point, the second equations in (4.2) hold identically in $\left(\xi^{2}, \xi_{j}^{k}\right)$ standing for the elements of $\mathbb{B}_{0}$. And hence $\operatorname{dim} G_{0}=n(n-1) / 2+1$. This, however, contradicts the fact that $G_{0}$ is a subgroup of the rotation group $S O(n)$. Therefore, $V$ vanishes nowhere under consideration, and consequently

$$
\operatorname{dim} G_{0}=\frac{1}{2}(n-1)(n-2) \text {. }
$$

Thus, we have

THEOREM 4.1. Lie algebras of the infinitesimal isometries preserving a nonzero vector field are of dimension $n(n-1) / 2+1$ at most. The preserved vector field never vanishes, and a maximal dimensional algebra, when such exists, is transitıve.

In what follows we study structures of Riemannian spaces admitting maximal Lie algebras preserving a non-zero vector field. Incidentally, a Riemannian space which admits an isometry group of dimension $n(n-1) / 2+1$ have been already studied by Yano [9] for $n>4(n \neq 8)$ and by Obata [10] for $n=8$. Homogeneous Riemannian spaces of dimension three and four have been studied by Cartan [11] and Ishihara [12], respectively. In case of $n=2$ and $\operatorname{dim} \$=2$, we go back to Bianchi (see [13], for instance).

On the basis of their results we can determine preserved vector fields (i. e., vector fields commutative with any element of (S) in each case. The notations will be fixed as above.

(i) $n=2$. If $\&$ is Abelian, $M$ is locally flat. A basis of $\&$ and a preserved vector field $V$ are given, respectively, by

$$
\begin{gathered}
X_{1}=\frac{\partial}{\partial x}, \quad X_{2}=\frac{\partial}{\partial y}, \\
V=a \frac{\partial}{\partial x}+b \frac{\partial}{\partial y} \quad(a, b: \text { constants }) .
\end{gathered}
$$

If $(3)$ is non-Abelian, $M$ is a space of constant negative curvature [13]. A basis of $\mathbb{B}$ is given in a suitably chosen coordinate system by 


$$
X_{1}=e^{-y} \frac{\partial}{\partial x}, \quad X_{2}=\frac{\partial}{\partial y} .
$$

A vector field commutative with $X_{1}$ and $X_{2}$ turns out to be

$$
V=(-b x+a) \frac{\partial}{\partial x}+b \frac{\partial}{\partial y},
$$

where $a$ and $b$ are constants. Thus we have

THEOREM 4.2. Let $M$ be a two-dimensional Riemannian space which admits a maximal two-dimensional Lie algebra of the infinitesimal isometries preserving a non-zero vector field. Then $M$ is locally flat or of constant negative curvature. The preserved vector field is given in a suitable coordinate system by (4.6) or (4.8) according as $M$ is flat or of constant negative curvature.

(ii) $n=3$. In this case the maximal dimension of $\mathbb{B}$ is equal to four. Riemannian spaces of dimension three admitting four-dimensional isometry groups are classified into two types [11]. One is defined in a suitable coordinate system by the metric

$$
d s^{2}=\frac{B}{z^{2}}\left(d x^{2}+d y^{2}+d z^{2}\right) \quad(B>0: \text { constant })
$$

and the other by the metric

$$
\begin{aligned}
d s^{2}=A \frac{d x^{2}+d y^{2}}{\left(1+\frac{K}{4}\left(x^{2}+y^{2}\right)\right)^{2}}+\left(d z+B \frac{x d y-y d x}{1+\frac{K}{4}\left(x^{2}+y^{2}\right)}\right)^{2} \\
(A>0, B: \text { consts., } K=-1,0,+1) .
\end{aligned}
$$

The preserved vector field can be easily written as

$$
V=c z \frac{\partial}{\partial z} \quad(c: \text { constant })
$$

in the first case (4.9) and as

$$
V=c^{\prime} \frac{\partial}{\partial z} \quad\left(c^{\prime}: \text { constant }\right)
$$

in the second case (4.10). Thus we obtain

THEOREM 4.3. Let $M$ be a three-dimensional Riemannian space which admits a maximal four-dimensional Lie algebra of infinitesimal isometries preserving a non-zero vector field. Then $M$ has a metric defined by either (4.9) or (4.10). The preserved vector field is given by (4.11) or (4.12) according to whether the metric is given by (4.9) or (4.10). 
(iii) $n \geqq 4$. The maximal dimension of $\mathbb{B}$ is equal to $n(n-1) / 2+1$. An isotropy subgroup $G_{0}$, isomorphic to a subgroup of $\mathrm{SO}(n)$, plays a key role to the problem, where $\operatorname{dim} G_{0}=(n-1)(n-2) / 2$ as is given by (4.4). Incidentally, we know a lemma about subgroups of dimension $(n-1)(n-2) / 2$ of $\mathrm{SO}(n)$ [10], [12], which is stated as follows.

LEMMA 4.4. If $n \neq 4,8$, then $G_{0}$ is isomorphic to $\mathrm{SO}(n-1)$ leaving a onedimensional subspace of $\boldsymbol{R}^{n}$ invariant. If $n=4, G_{0}$ is isomorphic to either $\mathrm{SO}(3)$ having a one-dimensional invariant subspace of $\boldsymbol{R}^{4}$ or $\mathrm{SU}(2)$ as the real representation in $\mathrm{GL}(4, \mathbf{R})$. If $n=8, G_{0}$ is isomorphic to either $\mathrm{SO}(7)$ having a onedimensional invariant subspace of $\boldsymbol{R}^{8}$ or Spin(7) acting irreducibly on $\boldsymbol{R}^{8}$.

Since SU(2) acting on $\boldsymbol{R}^{4}$ and Spin(7) acting on $\boldsymbol{R}^{8}$ have no invariant subspaces of $\boldsymbol{R}^{4}$ and of $\boldsymbol{R}^{8}$ respectively, any vector field commutative with the elements of $\mathfrak{G}_{0}$ (the Lie algebra of $\mathrm{SU}(2)$ or Spin(7) acting on a tangent space to $M$ ) vanishes at every point. Thus, it suffices for us to deal with only the case $G_{0}=\mathrm{SO}(n-1)$ leaving a one-dimensional subspace of $\boldsymbol{R}^{n}(n \geqq 4)$ invariant. Through the same argument as in [9], $M$ turns out to be a Riemannian space endowed with a metric locally reducible to either

$$
\begin{gathered}
d s^{2}=\left(d x^{1}\right)^{2}+g_{\lambda \mu} d x^{2} d x^{\mu} \quad \text { or } \\
d s^{2}=\frac{\sum\left(d x^{i}\right)^{2}}{k^{2}\left(x^{1}\right)^{2}} \quad(k \neq 0: \text { constant }),
\end{gathered}
$$

where $g_{\lambda \mu} d x^{\lambda} d x^{\mu}$ defines an $(n-1)$-dimensional Riemannian space of constant curvature. In accordance with (4.13) or (4.14), a basis of $(3$ consists of

$$
\begin{gathered}
\frac{\partial}{\partial x^{1}}, \quad \xi^{\lambda} \frac{\partial}{\partial x^{\lambda}} \text { or } \\
x^{2} \frac{\partial}{\partial x^{2}}, \quad x^{\lambda} \frac{\partial}{\partial x^{\mu}}-x^{\mu} \frac{\partial}{\partial x^{\lambda}}, \quad \frac{\partial}{\partial x^{\nu}},
\end{gathered}
$$

where $\xi^{\lambda} \partial / \partial x^{\lambda}$ stands for a basis of all the Killing vector fields on an $(n-1)$ dimensional Riemannian space of constant curvature.

To solve Eqs. (4.1) and (4.2), we need only to determine vector fields $V$ commutative with any element of (4.15) or (4.16). It is, however, an easy matter. We obtain then

$$
V=c \frac{\partial}{\partial x^{1}} \quad(c \neq 0: \text { constant })
$$

for $\mathbb{B}$ given by (4.15) and

$$
V=c^{\prime} x^{1} \frac{\partial}{\partial x^{1}} \quad\left(c^{\prime} \neq 0: \text { constant }\right)
$$

for (S given by (4.16). Thus we have 
THEOREM 4.5. Let $M$ be an $n$-dimensional ( $n \geqq 4$ ) Riemannian space which admits a maximal Lie algebra of infinitesimal isometries preserving a non-zero vector field. Then $M$ has a metric locally reducible to either (4.13) or (4.14). The preserved vector field is given by (4.17) or (4.18) according as the metric is of the form (4.13) or (4.14).

This theorem can be set up in global situation. Let $M$ be an $n$-dimensional Riemannian manifold, $I(M)$ the group of isometries of $M$, and $G$ a closed connected subgroup of $I(M)$. It is known today [16] what Riemannian manifolds can admit the subgroups $G$ of dimension $n(n-1) / 2+1$. According to [16], such a Riemannian manifold $M(n \geqq 5)$ is one of the following:

(1) $\quad M=\boldsymbol{R} \times M_{n-1}$, where $M_{n-1}$ is a complete simply connected space of constant curvature, and $G=\boldsymbol{R} \times I^{0}\left(M_{n-1}\right)$;

(2) $\quad M=S^{1} \times M_{n-1}$, where $M_{n-1}$ is as above and $G=S^{1} \times I^{0}\left(M_{n-1}\right)$;

(3) $\quad M=\boldsymbol{R} \times P_{n-1}(\boldsymbol{R})$ and $G=\boldsymbol{R} \times I^{0}\left(P_{n-1}(\boldsymbol{R})\right)$;

(4) $\quad M=S^{1} \times P_{n-1}(\boldsymbol{R})$ and $G=S^{1} \times I^{0}\left(P_{n-1}(\boldsymbol{R})\right)$;

(5) $M$ is a simply connected homogeneous Riemannian manifold $G / H$ with $H=\mathrm{SO}(n-1)$. It admits a $G$-invariant unit vector field $V$ and a $G$-invariant Riemannian metric of constant negative curvature (which agrees with the originally given metric on the tangent vectors perpendicular to $V$ );

(6) $M=\boldsymbol{R}^{8}$ and $G=\boldsymbol{R}^{8} \cdot \operatorname{Spin}(7)$.

From the viewpoint of vector-preserving isometry, among the list of Riemannian manifolds shown above (1) to (5) are possible. The preserved vector field $V$ is the infinitesimal transformation of a one-parameter subgroup $\boldsymbol{R}$ or $S^{1}$ of $G$ for each case (1) to (4). For the case (5) the preserved vector field $V$ has been already given above. Needless to say, these preserved vectors never vanish (see also Theorem 4.1).

\section{$\S 5$. Scalar-preserving isometries.}

A dynamical system $(T(M), Z$ ) is called simple (or conservatıve), if there is a function $U$ on $M$, referred to as a potential, such that

$$
V=-\operatorname{grad} U \text {. }
$$

We are going to specialize in the infinitesimal isometries preserving (5.1), and to characterize the gradient field (5.1). Eqs. (2.7) with $V$ satisfying (5.1) imply that $\mathcal{L}_{X} d U=d(X U)=0$, so that $X U=$ constant. To consider a subalgebra of $\mathbb{S}$ treated in Section 4, we impose the condition that $X U=0$. Then (2.7) is reduced to

$$
\mathcal{L}_{X} g_{\imath \jmath}=0, \quad X U=0 .
$$


Though these equations was studied by Ikeda and Nishino [1], here we have to supplement their results. Eqs. (5.2) can be put into a normal form:

$$
\begin{gathered}
\nabla_{\imath} \xi^{\jmath}=\xi_{i},, \quad \nabla_{k} \xi_{i} \cdot=-R_{l k \imath}{ }^{\jmath} \xi^{l}, \\
\xi_{\imath \jmath}+\xi_{j i}=0, \quad \xi^{\imath} U_{i}=0,
\end{gathered}
$$

where $U_{\imath}=\partial U / \partial x^{\imath}$. The integrability conditions of the second equation of (5.4) are given by

$$
\xi^{i} \nabla_{\imath} U_{\jmath}+\xi_{k \imath} \delta_{\jmath}^{k} U^{i}=0 .
$$

We now show that (5.5) is independent of (5.4). In fact, if not, there are functions $A$, such that

$$
\xi^{i} \nabla_{\imath} U_{\jmath}+\xi_{k \imath} \delta_{j}^{k} U^{i}=A_{j} \xi^{\imath} U_{\imath}
$$

hold identically in $\left(\xi^{2}, \xi_{j k}\right)$ satisfying the first equations in (5.4). This means that $\delta_{j}^{k} U^{i}=\delta_{j}^{\imath} U^{k}$. By contacting them for $\imath$ and $j$, we have $(n-1) U^{k}=0$, hence $U^{k}=0$, contradicting the assumption that $V=-\operatorname{grad} U$ is not the zero. We mean by $\mathfrak{h}$ the Lie algebra of the infinitesimal transformations satisfying (5.2). Like Theorem 4.1, we can prove the following theorem which was first proved in [14].

THEOREM 5.1. Lie algebras of the infinitesimal isometnes preserving a nonconstant scalar are of dimension $n(n-1) / 2$ at most, and are intransitive.

Let $H$ be a local isometry group generated by a maximal Lie algebra $\mathfrak{h}$ and $H_{0}$ an isotropy subgroup of $H$ at a point of $M$. Then $\operatorname{dim} H_{0}$ satisfies

$$
\frac{1}{2}(n-1)(n-2)-1 \leqq \operatorname{dim} H_{0} \leqq \frac{1}{2} n(n-1) \text {. }
$$

If $\operatorname{grad} U=0$ at a point, (5.5) and the second equation of (5.4) are identically valid at the point for $\left(\xi^{2}, \xi_{j k}\right)$ corresponding to the elements of the Lie algebra $\mathfrak{h}_{0}$ of $H_{0}$. Consequently we conclude that

$$
\operatorname{dim} H_{0}=\frac{1}{2} n(n-1)
$$

at a point where $\operatorname{grad} U=0$. Since $\mathfrak{h}$ is intransitive, $\operatorname{dim} H_{0} \neq(n-1)(n-2) / 2-1$. Therefore, at each point where $\operatorname{grad} U \neq 0$ we have for $n \neq 4$

$$
\operatorname{dim} H_{0}=\frac{1}{2}(n-1)(n-2),
$$

because $H_{0}$ is a closed subgroup of $\mathrm{SO}(n)$ and because $\mathrm{SO}(n)(n \neq 4)$ has no proper closed subgroups of dimension greater than $(n-1)(n-2) / 2$ [15]. The case where (5.7) holds was out of consideration in [1]. 
As such, according as $\operatorname{grad} U$ vanishes at a point or not, there occur following two cases to be considered: the case (A) where (5.7) holds at some point of $M$, and the case (B) where (5.8) holds everywhere.

We begin with the case (A).

THEOREM 5.2. If an $n$-dimensional Riemannian space $M(n \geqq 3)$ admits a Lie algebra of dimension $n(n-1) / 2$ consisting of the infinitesimal isometries preserving a non-constant scalar and if the dimension of an isotropy subgroup satısfies (5.7) at some point of $M$, then a metric $d s^{2}$ and a potential $U$ are given in a suitable coordinate system by

$$
\begin{gathered}
d s^{2}=A(r) \sum\left(d x^{i}\right)^{2}+B(r)\left(\sum x^{2} d x^{i}\right)^{2}, \\
U=U(r),
\end{gathered}
$$

where $r^{2}=\sum\left(x^{i}\right)^{2}$, and $A(r)$ and $B(r)$ are functions of $r \geqq 0$.

Proof. Choose such a coordinate system $\left(x^{i}\right)$ that any element $\phi$ of $H_{0}$ acts on $M$ in the form $\phi^{2}(x)=c^{2}, x^{\jmath}$ with $\left(c^{2},\right) \in \mathrm{SO}(n)$. After an analogous method as in $[1](n \geqq 3)$, we can obtain (5.9) on account of the equations

$$
g_{k l}(p)=g_{\imath j}(\phi(p)) c_{k}{ }_{k} c^{j}{ }_{l}
$$

which verify that $\phi$ is an isometry in the present coordinate neighborhood, where $p$ is an arbitrary point. In the present coordinate system a Killing vector field $X$ is of the form

$$
X=\nu_{i}{ }^{\jmath} x^{2} \frac{\partial}{\partial x^{j}},
$$

where $\left(\nu_{i}^{j}\right)$ are constants such that $\nu_{i}^{j}+\nu_{j}^{2}=0$. The second equation in (5.2) with $X$ replaced by (5.11) implies (5.10). This is because $\mathrm{SO}(n)$ acts transitively on each sphere of radius $r$. Needless to say, $\operatorname{grad} U$ must vanish at $r=0$. This completes the proof.

We now proceed to the case (B). The following theorem was proved in [1].

THEOREM 5.3. If an $n$-dimensional Riemannian space $M(n \geqq 4)$ admits a Lie algebra of dimension $n(n-1) / 2$ consisting of the infinitesimal isometries preserving a non-constant scalar and if the dimension of an isotropy subgroup satisfies (5.8) everywhere, then $M$ has a metric $d s^{2}$ and a potential $U$ locally reducible to

$$
d s^{2}=(d t)^{2}+f(t) d \sigma^{2},
$$

$$
U=U(t)
$$

where $f(t)$ is a positive function of $t, t$ being a parameter of $\boldsymbol{R}$, and $d \sigma^{2}$ is a metric defining an $(n-1)$-dimensional Riemannan space of constant curvature. 
Riemannian spaces admitting isometry groups of dimension $n(n-1) / 2$ have been studied globally in [17] without reference to potential functions. Let $G$ be a connected closed subgroup of $I(M)$, consisting of all the isometries leaving a non-constant function $U$ invariant. Manifestly $G$ is intransitive and $\operatorname{dim} G=$ $n(n-1) / 2$ (see Theorem 5.1). According to [17] a Riemannian manifold $M(n \geqq 6)$ admitting such a subgroup must be one of the following:

(1) $M$ is diffeomorphic to $\boldsymbol{R}^{n}, S^{n}$ or $P_{n}(\boldsymbol{R})$. The group $G$ has a common fixed point and $G=\mathrm{SO}(n)$. The Riemannian structure of $M$ is a one which is invariant under the action of $G$.

(2) $M=\left(S^{n-1} \times \boldsymbol{R}\right) / \Gamma$, where $\Gamma$ is a discrete group acting freely. The possibilities for $\Gamma$ are $\{1\}, Z_{2}, Z$ and $Z_{2} \times Z$. The group $G$ comes from the isometry group $I^{0}\left(S^{n-1}\right)$ of the $(n-1)$-dimensional factor in the covering space $S^{n-1} \times \boldsymbol{R}$.

(3) $M$ is non-compact and foliated by closed surfaces which are either all isometric to $\boldsymbol{R}^{n-1}$ or all isometric to hyperbolic spaces (i. e., complete simply connected spaces of constant negative curvature). These leaves are the $G$-orbits. If $M$ is complete, $M$ is diffeomorphic to $\boldsymbol{R}^{n}$ or $S^{1} \times \boldsymbol{R}^{n-1}$.

Without the restriction that $G$ is intransitive, allowed are other Riemannian manifolds which are of no interest for us. The preserved scalar $U$ is a one such that it is constant on each $G$-orbit. This closes the remark on global situation.

Changing situations, we assume that a Lie algebra of the infinitesimal 1sometries preserving a non-zero vector field $V$ is of dimension $n(n-1) / 2$ without assuming $V=-\operatorname{grad} U$. Let $\mathfrak{h}^{\prime}$ be a Lie algebra of dimension $n(n-1) / 2$ determined by (2.7), $H^{\prime}$ a local isometry group generated by $\mathfrak{h}^{\prime}$, and $H_{0}{ }^{\prime}$ the isotropy subgroup of $H^{\prime}$ at a point of $M$. Then $\operatorname{dim} H_{0}^{\prime}$ also satisfies (5.6). On the same argument used above, we have the following cases:

$$
\operatorname{dim} H_{0}^{\prime}=\frac{1}{2} n(n-1),
$$

$$
\operatorname{dim} H_{0}{ }^{\prime}=\frac{1}{2}(n-1)(n-2),
$$

$$
\operatorname{dim} H_{0}{ }^{\prime}=\frac{1}{2}(n-1)(n--2)-1
$$

We first assume $(5.14 \mathrm{c})$ which occurs when and only when $H^{\prime}$ is transitive. It is known that for $n \geqq 5 H_{0}^{\prime}$ with the dimensionality given by $(5.14 \mathrm{c})$ is transitive on the unit sphere $S^{n-1}$ in the tangent space to $M$ at the present point [17]. Accordingly, $H_{0}{ }^{\prime}$ has no invariant subspaces of the tangent space, and hence the vector field preserved by $\mathfrak{h}_{0}{ }^{\prime}$ must vanish at that point. Because of the transitivity of $H^{\prime}, V$ vanishes also in a neighborhood of the point, which contradicts the assumption that $V$ has only isolated zero points. Thus the case (5.14c) does not occur for $n \geqq 5$. 
As such there remain the two cases (5.14a) and (5.14b). These cases, however, coincide with (5.7) and (5.8), so that Theorem 5.2 and 5.3 can be recovered except for the statement of potentials. It is easily seen that in these cases $H^{\prime}$ is intransitive and hence there is a family of $(n-1)$-dimensional subspaces of $M$ invariant under the group actions. From this a new condition follows: $X U=0$, where $X$ is an arbitrary infinitesimal transformation of $\mathfrak{h}^{\prime}$ and $U$ is such a scalar that $U=$ constant defines an invariant subspace of $M$. Here we have recovered (5.2).

The gradient field grad $U$ is, of course, preserved by $X$ because of $X U=0$, but the question now arises as to whether or not the vector field $V$ preserved by infinitesimal isometries is a gradient field. Let $U$ be the scalar introduced above. Define a function $f$ by

$$
V^{j} U_{\jmath}=f U^{j} U_{\jmath}
$$

where $\left(V^{i}\right)$ and $\left(U^{i}\right)$ are local components of $V$ and $\operatorname{grad} U$ respectively. We break up $V$ into two fields as

$$
V=f \operatorname{grad} U+W \text {. }
$$

By differentiating $U$ with respect to $V$ and by the definition (5.15) of $f$, we find that $W=\left(W^{i}\right)$ is tangent to every hypersurface $U=$ constant:

$$
W^{i} U_{i}=0 .
$$

Let $X$ be an infinitesimal isometry belonging to $\mathfrak{h}^{\prime}$. We can show that $V^{i} U_{\imath}$ and $U^{i} U_{\imath}$ are both left invariant by $X$ :

$$
X\left(V^{i} U_{\imath}\right)=X\left(U^{i} U_{\imath}\right)=0 .
$$

In fact, by appealing to the equations $[X, V]=0$ and $d(X U)=0$, that is, $\xi^{k} \nabla_{k} V^{i}-V^{k} \nabla_{k} \xi^{\imath}=0$ and $\xi^{k} \nabla_{k} U_{\imath}+U_{k} \nabla_{\imath} \xi^{k}=0$, respectively, and by straightfoward calculation, we have

$$
\begin{gathered}
X\left(V^{i} U_{\imath}\right)=V^{k} \nabla_{k} \xi^{\imath} U_{\imath}-V^{i} U_{k} \nabla_{\imath} \xi^{k}=0, \\
X\left(U^{i} U_{\imath}\right)=2 \xi^{k} \nabla_{k} U_{i} U^{i}=-\left(\nabla_{i} \xi_{k}+\nabla_{k} \xi_{\imath}\right) U^{k} U^{i}=0 .
\end{gathered}
$$

This proves (5.18). From (5.15) and (5.18) we obtain

$$
X f=0 .
$$

Since $X$ of $\mathfrak{h}^{\prime}$ preserves $V$ given by (5.16), it follows from (5.19) and $d(X U)=0$ that

$$
[X, W]=0 .
$$


Next we show that $W=0$. Since $X$ is thought of as a Killing vector field on each hypersurface $U=$ constant [1] and since $W$ is tangent to the hypersurface (see (5.17)), $\mathfrak{h}^{\prime}$ is regarded as a Lie algebra of the infinitesimal isometries preserving a vector field $W$ on each $(n-1)$-dimensional hypersurface $U=$ constant (see (5.20)). Consequently Theorem 4.1 applied to $\mathfrak{h}^{\prime}$ implies that $W=0$. Thus we obtain

$$
V=f \operatorname{grad} U
$$

Choose the Gauss coordinate system adapted for a family of hypersurfaces $U=$ constant :

$$
d s^{2}=(d t)^{2}+g_{\lambda \mu}\left(t, x^{\kappa}\right) d x^{\lambda} d x^{\mu}, \quad U=U(t) .
$$

Then the function $f$ introduced in (5.15) becomes a function of $t$ alone because of (5.19). Introduce a function $\bar{U}(t)$ by $\bar{U}(t)=-\int(f(t) d U / d t) d t$. We then see from (5.21) that $V=(-d \bar{U} / d t, 0, \cdots, 0)$ in the present coordinate system. Therefore, we attain

$$
V=-\operatorname{grad} \bar{U}
$$

Summarizing the above discussion, we have

THEOREM 5.4. If an $n$-dimensional Riemannian space $M(n \geqq 5)$ admits a Lie algebra of dimension $n(n-1) / 2$ consisting of the infinitesimal isometries preserving a non-zero vector field, then the preserved vector field is a gradient field.

The author wishes to express his gratitude to Professor S. Ishihara for carefully reading the manuscript and offering valuable comments. The author is also grateful to Professor M. Ikeda for his encouragement.

\section{REFERENCES}

[1] M. IKEDA and Y. Nishino, On groups of scalar-preserving isometries in Riemannian spaces, with applications to dynamical systems, Tensor, N. S., 27 (1973), 295-305.

[2] G.H. Katzin and J. Levine, Related first integral theorem - A method for obtaining conservation laws of dynamical systems with geodesic trajectries in Riemannian spaces admitting symmetries, J. Math. Phys., 9 (1968), 8-15.

[3] G.H. KATzIN, Related integral theorem. II. A method for obtaining quadratic constants of the motion for conservative dynamical systems admitting symmetries, J. Math. Phys., 14 (1973), 1213-1217.

[4] J. Levine and G.H. Katzin, Symmetry mappings of constrained dynamical systems and an associated related integral theorem, J. Math. Phys., 14 (1973), 1886-1891. 
[5] G.H. Katzin and J. Levine, Dynamical symmetries and constants of the motion for classical particle systems, J. Math. Phys., 15 (1974), 1460-1470.

[6] T. IWAI, On kınematical invariances of the equations of motion, Internat. J. Theoret. Phys., 12 (1975), 383-392.

[7] M. IKeda and K. Sakamoto, On the concept of symmetry in Pontryagin's maxımum principle, SIAM J. Control, 13 (1975), 389-399.

[8] H.C. WANG and K. YANO, A class of affinely connected spaces, Trans. Amer. Math. Soc., 80 (1955), 72-96.

[9] K. YaNo, On $n$-dimensional Riemannian spaces admittıng a group of motions of order $n(n-1) / 2+1$, Trans. Amer. Math. Soc., 74 (1953), 260-279.

[10] M. OватA, On $n$-dimensional homogeneous spaces of Lie groups of dimension greater than $n(n-1) / 2$, J. Math. Soc. Japan, 7 (1955), 371-388.

[11] E. Cartan, Leçons sur la géométrie des espaces de Riemann, 2nd ed., Parıs, Gauther-Villars (1946).

[12] S. Ishihara, Homogeneous Riemannain spaces of four dimensions, J. Math. Soc. Japan, 7 (1955), 345-370.

[13] L.P. Eisenhart, Continuous groups of transformations, Princeton, Princeton Univ. Press (1933).

[14] M. IKEDA and T. Fujitani, On linear first integrals of natural systems in classical mechanics, Math. Japan., 15 (1971), 143-153.

[15] D. Montgomery and H. Samelson, Transformation groups of spheres, Ann. of Math., 44 (1943), 454-470.

[16] S. Kobayashi, Transformation groups in differential geometry, New York, Springer-Verlag (1972).

[17] S. Kobayashi and T. NAGano, Riemannian manifolds with abundant isometries, Differential geometry, in honor of K. Yano, Tokyo, Kinokuniya (1972), 195-219.

FACULTY OF ENGINEERING

Gifu University

NAKA-MACHI, Kagamigahara

GIFU, JAPAN 